\section{(6) OPEN ACCESS}

\title{
Non-superiority of lumen-apposing metal stents over plastic stents for drainage of walled-off necrosis in a randomised trial
}

\author{
Ji Young Bang, Udayakumar Navaneethan, Muhammad K Hasan, Bryce Sutton, \\ Robert Hawes, Shyam Varadarajulu
}

Additional material is published online only. To view, please visit the journal online (http://dx.doi.org/10.1136/ gutjnl-2017-315335).

Center for Interventional Endoscopy, Florida Hospital, Orlando, Florida, USA

Correspondence to Dr Shyam Varadarajulu, Center for Interventional Endoscopy, Florida Hospital, Orlando, FL 32803, USA;

svaradarajulu@yahoo.com

Oral presentation, Pancreas Club 2017, Chicago, Illinois, USA.

Received 22 September 2017 Revised 9 April 2018 Accepted 2 May 2018 Published Online First 1 June 2018

\section{SLinked}

- http://dx.doi.org/10.1136/ gutjnl-2018-316994

Check for updates

To cite: Bang JY,

Navaneethan U, Hasan MK, et al. Gut

2019:68:1200-1209.

\section{ABSTRACT \\ Objective Although lumen-apposing metal stents} (LAMS) are increasingly used for drainage of walled-off necrosis (WON), their advantage over plastic stents is unclear. We compared efficacy of LAMS and plastic stents for WON drainage.

Design Patients with WON were randomised to endoscopic ultrasound-guided drainage using LAMS or plastic stents. Primary outcome was comparing total number of procedures to achieve treatment success defined as symptom relief in conjunction with WON resolution on CT at 6 months. Secondary outcomes were treatment success, procedure duration, clinical/stentrelated adverse events, readmissions, length of hospital stay (LOS) and costs.

Results 60 patients underwent LAMS ( $n=31)$ or plastic stent $(n=29)$ placement. There was no significant difference in total number of procedures performed (median 2 (range 2-7) LAMS vs 3 (range 2-7) plastic, $p=0.192$ ), treatment success, clinical adverse events, readmissions, LOS and overall treatment costs between cohorts. Although procedure duration was shorter (15 vs 40 min, $p<0.001)$, stent-related adverse events $(32.3 \%$ vs $6.9 \%, p=0.01$ ) and procedure costs (US\$12 155 vs US\$6609, $p<0.001)$ were higher with LAMS. Significant stent-related adverse events were observed $\geq 3$ weeks postintervention in LAMS cohort. Interim audit resulted in protocol amendment where CT scan was obtained at 3 weeks postintervention followed by LAMS removal if WON had resolved. After protocol amendment, there was no significant difference in adverse events between cohorts.

Conclusion Except for procedure duration, there was no significant difference in treatment outcomes between LAMS and plastic stents. To minimise adverse events with LAMS, patients should undergo follow-up imaging and stent removal at 3 weeks if WON has resolved. Trial registration number NCT02685865.

\section{INTRODUCTION}

Pancreatic fluid collections (PFCs) are broadly categorised as pseudocysts or walled-off necrosis (WON). Endoscopic treatment outcomes for pseudocyst drainage are superior because the fluid drains quickly and completely. ${ }^{12}$ On the other hand, endoscopic treatment outcomes for WON are only modest because the clearing of solid debris may be incomplete and can become

\section{Significance of this study}

What is already known on this subject?

- Lumen-apposing metal stents (LAMS) are being increasingly used for endoscopic ultrasound (EUS)-guided drainage of pancreatic fluid collections (PFC). However, the advantage of LAMS over plastic stents is not clear.

What are the new findings?

- This randomised trial showed that there was no significant difference in the number of procedures performed to achieve treatment success, clinical adverse events, readmissions, length of stay and overall treatment costs between LAMS and plastic stent cohorts in EUS-guided drainage of walled-off necrosis (WON).

- However, significantly higher rate of stentrelated adverse events was observed if the LAMS was not removed within 3 weeks postintervention.

How might it impact on clinical practice in the foreseeable future?

- As there is no significant difference in clinical outcomes between LAMS and plastic stents, the choice of stent used during EUS-guided WON drainage should be based on clinical status, pancreatic duct integrity and patient compliance. If LAMS are placed, they must be removed at 3 weeks postprocedure.

infected. $^{3} 4$ In a study of 211 patients who underwent endoscopic transmural drainage of PFCs, treatment success was significantly higher for pseudocysts compared with WON, 93.5\% vs $63.2 \%, \quad \mathrm{p}<0.00010 .{ }^{4}$ Consequently, several technical modifications have been proposed to improve WON drainage including dual modality treatment that combines endoscopic and percutaneous drainage, creation of multiple gateways (internal fistulae), direct transluminal or sinus tract necrosectomy and recently, placement of lumen-apposing metal stents (LAMS). ${ }^{5-8}$ It is postulated that the large diameter $(10-20 \mathrm{~mm})$ of LAMS facilitates better drainage of WON contents than the small calibre (7-10 Fr) plastic stents, 
thereby necessitating fewer number of procedures to achieve treatment success. Additionally, the risks of perforation and peritoneal leakage of PFC contents are less likely given the 'lumen-apposing' characteristic of the stent. However, LAMS are significantly more expensive than plastic stents and in the present era of healthcare, expensive technological advances must provide superior clinical outcomes if they increase cost. Published studies to date evaluating LAMS have been retrospective, registry-based, single-arm or non-comparative in design involving a heterogeneous patient subset. ${ }^{9-12}$

The primary objective of this randomised trial was to evaluate the effectiveness of LAMS by comparing the total number of procedures required to achieve treatment success between patients undergoing LAMS and plastic stent placement at 6-month follow-up. The secondary objectives were to compare treatment success, procedure duration, clinical and stent-related adverse events, readmissions, length of hospital stay (LOS) and treatment costs.

\section{METHODS}

\section{Participants}

After approval of the study by the Institutional Review Board at our institution, consecutive patients with WON were recruited from the inpatient ward service or outpatient referrals. All patients were evaluated with a contrast-enhanced CT or a MRI scan. WON was defined according to the revised Atlanta classification of acute pancreatitis as mature, encapsulated, liquefied necrosis located within the pancreatic parenchyma or peripancreatic space. ${ }^{13}$ All imaging studies were reviewed by an American Board Certified Radiologist with special expertise (fellowship-trained) in body imaging, who interprets 50-75 CT scans of the abdomen-pelvis per day. Written informed consents were obtained from all patients before enrolment in the study and the procedure selection criteria are shown in box 1 . All authors had full access to the study data and have reviewed and approved the final manuscript.

\section{Box 1 Selection criteria}

\section{Inclusion criteria}

1. Diagnosis of walled-off necrosis (WON) based on imaging criteria. $^{13}$

2. Documented history of acute pancreatitis.

3. Suspected or confirmed infected WON and/or symptomatic WON causing persistent pancreatic-type pain, with need for inpatient treatment using oral or intravenous narcotics at least three times a day.

4. WON identified at endoscopic ultrasound (EUS) and amenable for EUS-guided drainage.

\section{Exclusion criteria}

1. Age $<18$ years.

2. Pregnancy.

3. Contraindications to endoscopic drainage: Billroth II reconstruction, gastric bypass surgery, prior surgery for pancreas-related diseases.

4. WON not adherent to the GI wall or not accessible for endoscopic drainage.

5. Patients with indwelling percutaneous drainage catheters prior to randomisation.

\section{Randomisation and masking}

Patients were enrolled in this clinical trial by interventional endoscopists who evaluated the study subjects in the inpatient wards or preprocedure consultation rooms. Computer-generated randomisation assignments were provided by the statistician using a block randomisation method and placed in sequentially numbered, sealed, opaque envelopes that were opened by one of the study investigators intraprocedurally to determine the treatment allocation. Once the inclusion criteria were met, patients were randomised equally (1:1 allocation) to both treatment arms. Given the differences in the types of stents placed, endoscopists were not blinded to the treatment allocation.

\section{Procedural technique}

All interventions were performed using a therapeutic linear array echoendoscope under monitored anaesthesia care using propofol or general anaesthesia. Intravenous piperacillin/tazobactam or ciprofloxacin was administered in all patients prior to the drainage procedure. Interventions were undertaken by one of four endoscopists (SV, UN, MKH, RH) who are all experienced in the use of plastic stents for WON drainage, have individually performed > 20 LAMS placements and individually have a lifetime experience of performing $>25$ necrosectomy procedures.

\section{Lumen-apposing metal stents}

LAMS used in this study (Hot AXIOS stent and electrocautery-enhanced delivery system, Boston Scientific, Natick, Massachusetts, USA) were through-the-scope, fully covered self-expandable metal stents, $15 \mathrm{~mm}$ in diameter and $10 \mathrm{~mm}$ in length. After directly puncturing the WON using the electrocautery tip (without the use of a guidewire to assist in stent insertion), the delivery catheter was advanced into the fluid collection and the distal flange was deployed under endoscopic ultrasound (EUS) guidance. The proximal flange was then released under EUS guidance or endoscopic view (video 1).

\section{Plastic stents}

Double pigtail plastic stents (7 Fr $4 \mathrm{~cm}$ ) (Boston Scientific) were used in this study. The WON was first punctured using a 19-gauge $(\mathrm{G})$ fine-needle aspiration (FNA) needle and a 0.025 inch guidewire was coiled within the fluid collection under fluoroscopic guidance. After initial access using an endoscopic retrograde cholangiopancreatography (ERCP) cannula or cystotome, the tract was further dilated to $15 \mathrm{~mm}$ using a radial expansion balloon. Two plastic stents were then placed into the WON via the transmural fistula (video 2 ).

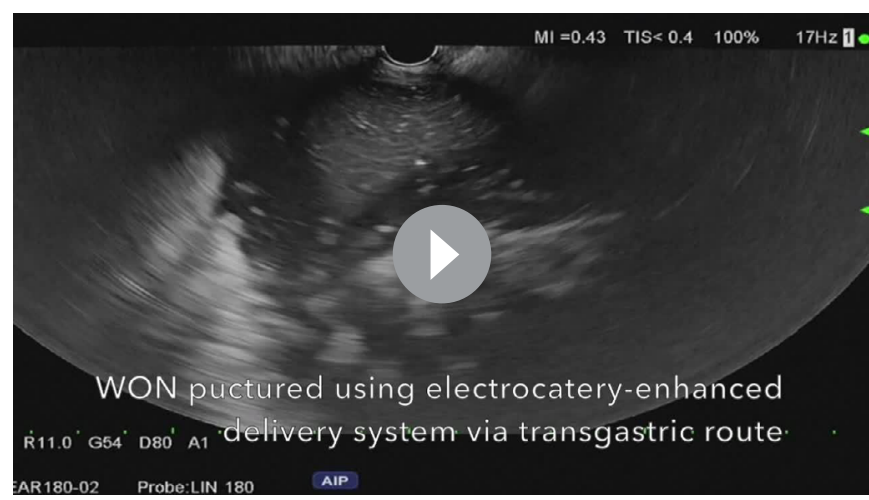

Video 1 Endoscopic ultrasound (EUS)-guided drainage of walled-off necrosis (WON) using lumen-apposing metal stents (LAMS). 


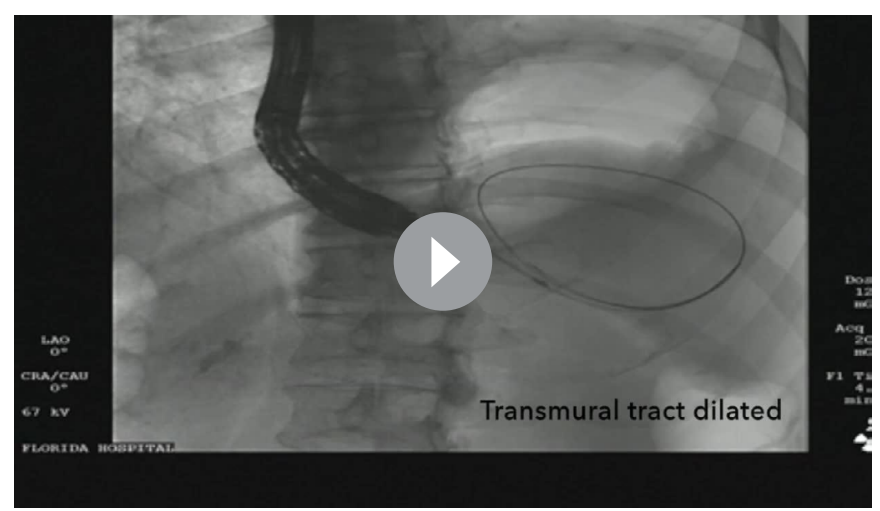

Video 2 Endoscopic ultrasound (EUS)-guided drainage of walled-off necrosis (WON) using plastic stents.

\section{Additional interventions}

In patients with WON measuring $>120 \mathrm{~mm}$ in size, two drainage tracts were created (multigate technique) using the same stent type if the drainage of necrotic contents was deemed inadequate for both stent groups. Additionally, nasocystic catheters (10 Fr) were inserted for lavage of necrotic cavity.

\section{Treatment algorithm}

All inpatients returned to the intensive care unit or hospital ward for ongoing care postprocedure and were discharged when their symptoms improved. Outpatients were admitted for 23 hours observation and discharged the next day unless persistently sick or if adverse events were encountered. At discharge, oral ciprofloxacin $500 \mathrm{mg}$ was prescribed to be taken twice daily for 5 days. For all patients with suboptimal treatment response as manifested by persistent or new-onset systemic inflammatory response syndrome (SIRS), a CT scan was obtained at 72 hours postintervention. If there was $<25 \%$ decrease in size of WON, additional interventions were undertaken after consultation with the pancreaticobiliary multidisciplinary team. If the WON comprised predominantly of liquefied debris, additional transmural tracts were created using the same stent type to facilitate better drainage. On the other hand, if the WON comprised predominantly of solid debris, then transluminal necrosectomy was performed using a single channel, cap-fitted therapeutic gastroscope. Necrosectomy comprised the following steps that were undertaken in sequence: debridement using oval/braided snares or rat-tooth forceps (video 3), extraction of necrotic debris using forceps, snares or retrieval nets (video 4) and irrigation using normal saline mixed with hydrogen peroxide (video 5).

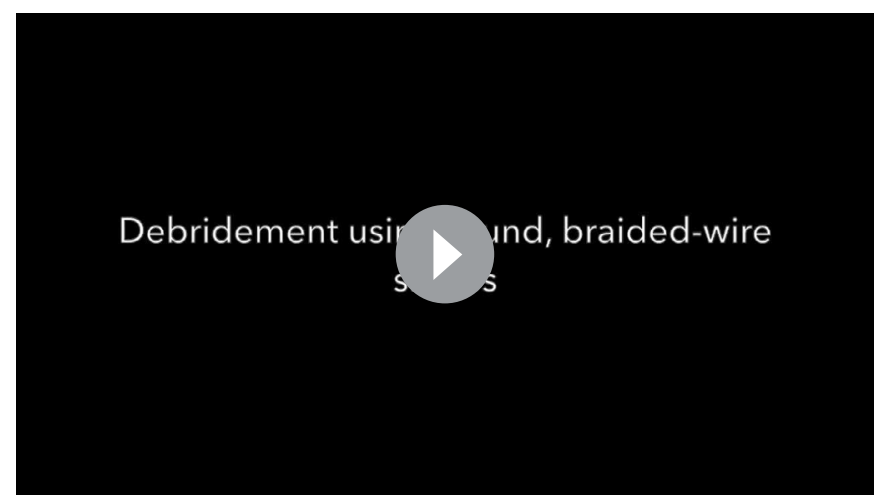

Video 3 Technique of endoscopic debridement using oval snares, braided-wire snares and forceps.

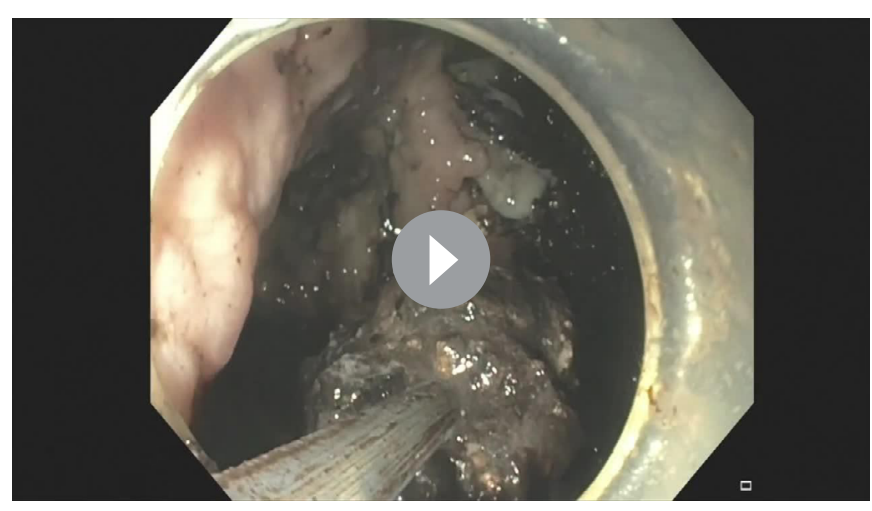

Video 4 Technique of extraction of necrotic debris.

\section{Patient follow-up}

On initial outpatient follow-up at 6 weeks postintervention, all patients underwent CT to assess treatment response prior to stent removal and an ERCP to assess for pancreatic duct integrity. All CT scans were reviewed by an expert radiologist. If WON had resolved and an intact main pancreatic duct was observed on ERCP, all transmural stents were removed by endoscopy. Patients in whom the WON had resolved but with disconnected pancreatic duct syndrome (DPDS), the plastic stents were left in situ indefinitely. ${ }^{14}$ In patients with DPDS who were treated with LAMS, the stent was exchanged for plastic stents to drain the upstream disconnected gland (video 6). Transpapillary pancreatic stents were placed in patient with partial disruption (but without disconnection) of the pancreatic duct.

At 3 and 6 months postprocedure, blinded outcomes assessment was performed by a research nurse coordinator who interviewed subjects at outpatient clinics or contacted them by telephone to obtain information on disease-related reinterventions and readmissions.

\section{Cost analysis}

All relevant costs related to treatment were considered including: procedure costs, inpatient hospital stay from date of procedure to discharge, pharmacy, anaesthesia, radiology and laboratory results. All costs were based on Medicare reimbursement fee structure and expressed in 2017 US dollars. To test for differences in total cost between stent types, generalised linear models (GLM) were used while adjusting for patient demographics, preintervention SIRS, preintervention organ failure, WON size, degree of necrosis and procedural technique. Prior to fitting the GLM, skewness of the total cost data was assessed and determined to be

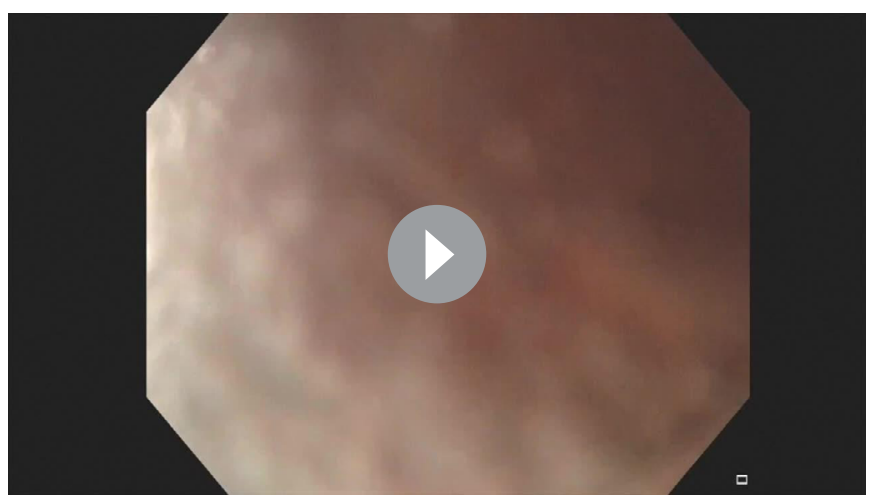

Video 5 Irrigation using normal saline mixed with hydrogen peroxide. WON, walled-off necrosis. 


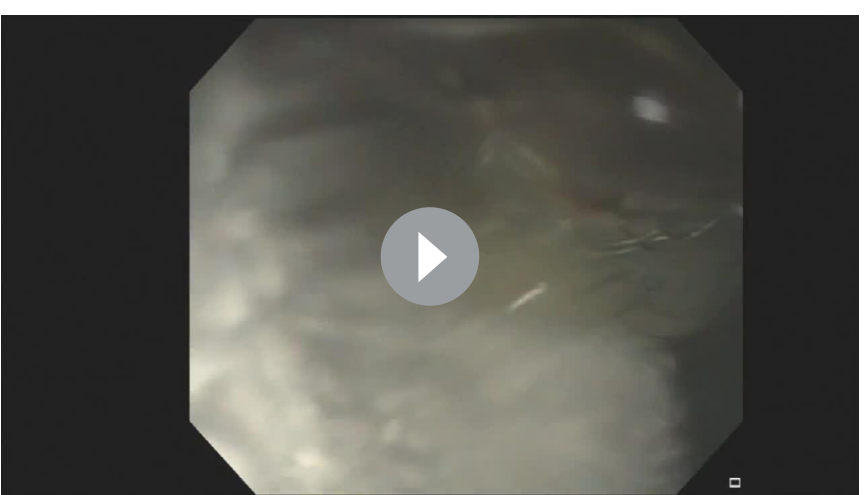

Video 6 Lumen-apposing metal stents (LAMS) is exchanged for plastic stents in a patient with walled-off necrosis (WON) and disconnected pancreatic duct syndrome. EUS, endoscopic ultrasound.

highly positively skewed. The Pregibon and modified Park tests indicated that the GLM should be fitted to the study data using a log link and gamma distribution for the mean-variance relationship of the response variable. ${ }^{15} 16$ Adjusted mean procedure and total cost estimates by stent type were calculated using the method of recycled predictions. ${ }^{17}$ While costs pertaining only to stent placement was reported as procedure costs, total costs included costs for the procedure, postprocedure hospitalisation, readmissions, pharmacy, anaesthesia, radiology, laboratory and other support. Further details on the cost analysis methodology are outlined in online supplementary table 1.

\section{Definitions}

The total number of procedures required to achieve treatment success included the index endoscopic intervention, endoscopic reinterventions, percutaneous drain placement, ERCP, enteral feeding tube placement and any procedures performed for the management of adverse events or disease-specific events in each cohort during the 6-month follow-up period. Technical success was defined as successful placement of LAMS or plastic endoprostheses within the WON. Treatment success was defined as resolution of WON on CT scan in association with clinical resolution of symptoms at 6-month follow-up. Treatment failure was defined as the need for rescue surgery or death due to underlying disease or intervention. Procedure duration was measured as the time from passage of the echoendoscope into the GI lumen to perform transmural drainage until the end of the endoscopic procedure. Length of stay (LOS) was defined as the time to hospital discharge from the day of index endoscopic intervention. Adverse events were classified per established criteria. ${ }^{18}$

\section{Outcome measures}

The main outcome measure was to compare the total number of procedures needed to achieve treatment success at 6-month follow-up. Secondary outcome measures were comparison of the treatment success, clinical and stent-related adverse events, resolution of preintervention SIRS, procedure duration, LOS, readmissions and overall treatment costs between cohorts.

\section{Sample size calculation}

A two-sided sample size calculation was performed to detect a difference of one in the total number of procedures needed to achieve treatment success (assuming one for LAMS and two for plastic stents), with SD of 0.7 for plastic stents and SD of 1.5 for LAMS (based on the available literature). ${ }^{19}{ }^{20}$ A power calculation performed using Stata statistical software (StataCorp, College Station, Texas, USA) at 90\% power and $\alpha=0.05$ resulted in sample size estimation at 58 patients ( 29 per cohort) and hence was set at 29 patients per treatment group.

\section{Statistical analysis}

Continuous data were summarised as means with SD or medians with IQR and range, and were compared using the Wilcoxon rank-sum test. Categorical data were summarised as frequencies with percentages and were compared using the $\mathrm{X}^{2}$ or the Fisher's exact test as indicated. A Poisson regression analysis was also performed to identify factors associated with the number of procedures performed for treatment success. In addition, a multiple linear regression analysis with adjustment for possible clustering of procedures within patients was further performed. All analyses were performed using the intention-to-treat principle. Statistical significance was determined at $\mathrm{p}<0.05$ and two-sided $\mathrm{p}$ values were reported for comparison of all outcome measures. All statistical analyses were performed using Stata V.14 (StataCorp).

A safety committee was appointed, comprising a surgeon, a nurse practitioner and a gastroenterologist who were not involved in the trial. The evolving results were monitored by the committee every 3 months.

\section{RESULTS}

\section{Study enrolment and termination}

Between February 2016 and March 2017, 80 patients were screened and 60 patients with WON underwent randomisation: 31 patients in the LAMS group and 29 patients in the plastic group (Figure 1). All patients were followed up for a minimum duration of 6 months. The patient and WON characteristics, and preprocedure details are shown in table 1.

\section{LAMS cohort}

Twenty-nine per cent of the LAMS cohort patients had preintervention SIRS or organ failure and the median degree of necrosis was 40\% (IQR 20) (tables 1 and 2). Drainage was undertaken using the multigate technique in three patients (9.7\%) and a mean of 2.8 procedures were needed to achieve treatment success of $93.5 \%$ (table 3 ). Additional procedures undertaken due to suboptimal treatment response at 72 hours (for ongoing SIRS) included creation of additional transmural tracts $(n=2)$, direct endoscopic necrosectomy $(n=4)$ and percutaneous drainage catheter placement $(n=2)$. The total number and details of individual procedures undertaken in the LAMS cohort for treatment success during the 6-month follow-up period are shown in table 3 and online supplementary table 2, respectively.

Stent-related adverse events were encountered in 10 of 31 (32.2\%) patients. Eight of 10 events were observed within the first 5 months from study commencement and included two patients in whom the stent was buried in the gastric wall, bleeding from the stent site in three and stent-induced biliary stricture in three patients. In two patients, the LAMS were found buried under the gastric mucosa on fluoroscopy when they presented for stent removal at 5 and 6 weeks postprocedure (figure $2 \mathrm{~A}, \mathrm{~B}$ ). In one patient, endoscopic stent retrieval resulted in massive bleeding requiring interventional radiology (IR)-guided coil embolisation. In the other patient, the buried LAMS was retrieved using biopsy forceps after transmural tract dilation. Three patients presented with severe GI bleeding requiring intensive care unit admissions and blood 


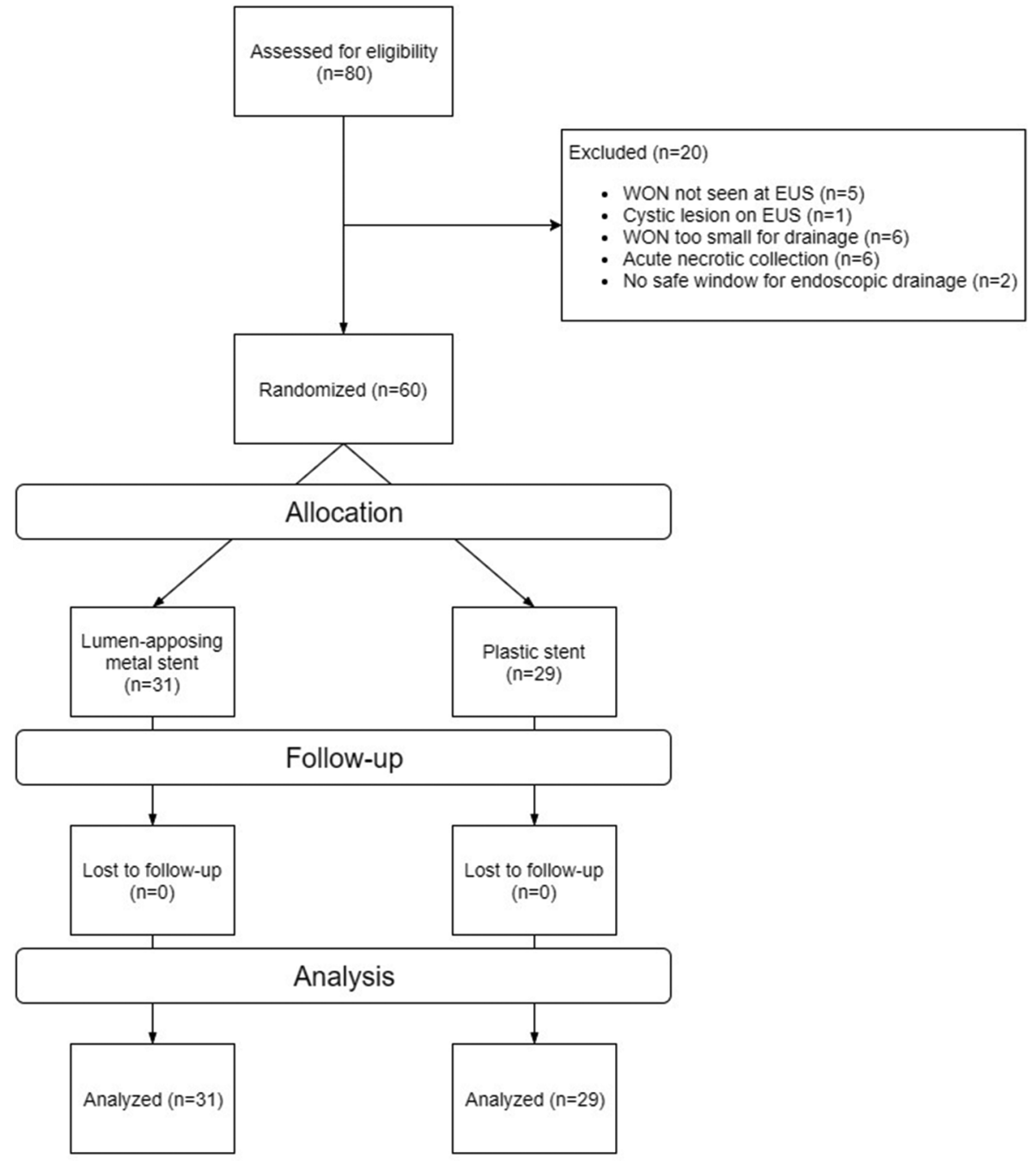

Figure 1 Consort flow diagram of patient enrolment. EUS, endoscopic ultrasound; WON, walled-off necrosis.

transfusions after $3(n=1)$ and 5 weeks $(n=2)$ poststent placement. On EUS examination, interlacing vessels were visualised within the distal flange of the LAMS (figure 3A). A CT angiogram confirmed pseudoaneurysms in all three patients (which were not previously visualised on preintervention cross-sectional imaging) that were managed by IR-guided coil embolisation. Three patients presented with obstructive jaundice at $5(n=1)$ and 6 weeks $(n=2)$ post-LAMS placement. At ERCP, a distal biliary stricture was observed secondary to mechanical compression induced by the LAMS that had been deployed via the duodenal bulb (figure 3B,C). The LAMS were removed and all patients were treated successfully by biliary sphincterotomy with or without temporary biliary stent placement.

All adverse events were observed in the LAMS cohort at 3 or more weeks postprocedure. Therefore, the safety board recommended an amendment to the study protocol whereby a CT was obtained at 3 weeks in the LAMS cohort followed by stent removal if the WON had resolved. Following a change to the study protocol, only two stent-related adverse events were observed. One involved LAMS migration into the GI tract and the other involved localised bleeding in the gastric mucosa when the WON was accessed with the tip of the electrocautery-enhanced delivery system. Stent migration into the GI tract manifested first as gastric outlet obstruction when the LAMS impacted the pylorus (figure 4A, B), then as small bowel obstruction when it impacted the jejunum (figure 4C) and then finally as faecal impaction when it occluded the rectosigmoid junction (figure 4D, E). The stent was retrieved at sigmoidoscopy using rat tooth forceps and no underlying colonic stricture was identified. The patient with gastric mucosal bleeding was managed endoscopically by injection of epinephrine and application of cautery.

Four clinical adverse events unrelated to LAMS placement were encountered in three patients. One patient developed cardiopulmonary arrest postprocedure and was successfully resuscitated. One patient in whom treatment was successful but with Child-Pugh class $\mathrm{C}$ cirrhosis developed fulminant liver failure and died awaiting liver transplantation. One patient was diagnosed with pulmonary embolism postprocedure and the percutaneous endoscopic gastrostomy (PEG) tube was also found to have traversed the left lobe of the liver on follow-up CT scan. The PEG tube was subsequently successfully removed endoscopically.

Pancreatography revealed a normal main pancreatic duct in 9 , DPDS in 13, partial duct disruption in 6 and the ductal status was unknown in 3 patients. Of the 13 patients with DPDS, a transmural plastic stent could not be placed in six patients after LAMS removal as the necrotic cavity had collapsed completely (video 7). A transpapillary pancreatic duct stent bridging the leak was placed in all patients with partial disruption, resulting in successful resolution of the leak. 


\begin{tabular}{|c|c|c|c|}
\hline & LAMS $(n=31)$ & Plastic $(n=29)$ & $P$ values \\
\hline \multicolumn{4}{|l|}{ Age (years) } \\
\hline Mean (SD) & $55.8(15.6)$ & $60.3(13.0)$ & 0.277 \\
\hline Median & 59 & 62 & \\
\hline IQR & 25 & 15 & \\
\hline Range & $22-83$ & $32-78$ & \\
\hline \multicolumn{4}{|l|}{ Gender: $\mathrm{n}(\%)$} \\
\hline Female & $11(35.5)$ & $13(44.8)$ & 0.460 \\
\hline Male & $20(64.5)$ & $16(55.2)$ & \\
\hline \multicolumn{4}{|l|}{ Ethnicity: n (\%) } \\
\hline Black & $3(9.7)$ & $1(3.4)$ & 0.750 \\
\hline Hispanic & $2(6.5)$ & $3(10.3)$ & \\
\hline White & $26(83.9)$ & $25(86.2)$ & \\
\hline \multicolumn{4}{|c|}{ Aetiology of acute pancreatitis: $n(\%)$} \\
\hline Alcohol & $9(29.0)$ & $5(17.2)$ & 0.453 \\
\hline Gallstones & $6(19.4)$ & $10(34.5)$ & \\
\hline Idiopathic & $12(38.7)$ & $12(41.4)$ & \\
\hline Other* & $4(12.9)$ & $2(6.9)$ & \\
\hline \multicolumn{4}{|c|}{ Serum white cell count $\left(x 10^{9}\right.$ cells/L) } \\
\hline Mean (SD) & $11.2(7.0)$ & $11.3(6.5)$ & 0.840 \\
\hline Median & 9.9 & 8.2 & \\
\hline IQR & 6.1 & 8.2 & \\
\hline Range & $2.9-33.0$ & $4.3-31.3$ & \\
\hline \multicolumn{4}{|l|}{ Serum albumin (g/dL) } \\
\hline Mean (SD) & $3.1(0.7)$ & $3.1(0.6)$ & 0.813 \\
\hline Median & 3.1 & 3.0 & \\
\hline IQR & 0.8 & 0.8 & \\
\hline Range & $1.8-4.4$ & $2.1-4.5$ & \\
\hline SIRS preprocedure: $n$ (\%) & $9(29.0)$ & $13(44.8)$ & 0.205 \\
\hline $\begin{array}{l}\text { Organ failure preprocedure: } \\
\mathrm{n}(\%)\end{array}$ & $2(6.5)$ & $4(13.8)$ & 0.417 \\
\hline \multicolumn{4}{|l|}{ Clinical presentation: $\mathrm{n}(\%)$} \\
\hline Infectiont & $27(87.1)$ & $26(89.7)$ & 0.999 \\
\hline Pain & $4(12.9)$ & $3(10.3)$ & \\
\hline \multicolumn{4}{|l|}{ ASA classification: $n(\%)$} \\
\hline II & $13(41.9)$ & $12(41.4)$ & 0.918 \\
\hline III & $17(54.8)$ & $15(51.7)$ & \\
\hline IV & $1(3.2)$ & $2(6.9)$ & \\
\hline $\begin{array}{l}\text { ICU or high-acuity unit care: } \\
\mathrm{n}(\%)\end{array}$ & $10(32.3)$ & $10(34.5)$ & 0.855 \\
\hline \multicolumn{4}{|c|}{ Route of nutrition at index intervention: $n(\%)$} \\
\hline Oral & $24(77.4)$ & $20(69.0)$ & 0.596 \\
\hline Enteral & $5(16.1)$ & $7(24.1)$ & \\
\hline TPN & $1(3.2)$ & $2(6.9)$ & \\
\hline None & $1(3.2)$ & 0 & \\
\hline
\end{tabular}

*Other aetiology of acute pancreatitis: in LAMS group—pancreatic cancer in one, pancreas divisum in one, post-ERCP pancreatitis in one, trauma in one patient. In plastic group-post-EUS-FNA in one, trauma in one patient.

tInfected WON defined as positive culture of aspirated contents or gas within WON seen on $\mathrm{CT}$.

ASA, American Society of Anesthesiologist; ERCP, endoscopic retrograde cholangiopancreatography; EUS, endoscopic ultrasound; FNA, fine- needle aspiration; ICU, intensive care unit; LAMS, lumen-apposing metal stent; SIRS, systemic inflammatory response syndrome; TPN, total parenteral nutrition.

\section{Plastic stent cohort}

Preintervention SIRS or multiorgan failure was observed in $44.8 \%$ of patients and the median degree of necrosis was $50 \%$ (IQR 20) (tables 2 and 3). Drainage was undertaken using the multigate technique in nine patients $(31.0 \%)$ and a mean of
Table 2 WON characteristics and procedural details

\begin{tabular}{|c|c|c|c|}
\hline & $\begin{array}{l}\text { LAMS } \\
(\mathrm{n}=31)\end{array}$ & $\begin{array}{l}\text { Plastic } \\
(n=29)\end{array}$ & $P$ values \\
\hline \multicolumn{4}{|l|}{ WON location: $n(\%)$} \\
\hline Head/uncinate & $9(29.0)$ & $6(20.7)$ & 0.456 \\
\hline Body/tail & $22(71.0)$ & $23(79.3)$ & \\
\hline \multicolumn{4}{|l|}{ Degree of necrosis (\%): } \\
\hline Mean (SD) & $40.3(15.7)$ & $45.3(14.4)$ & 0.173 \\
\hline Median & 40 & 50 & \\
\hline IQR & 20 & 20 & \\
\hline Range & $20-70$ & $20-80$ & \\
\hline \multicolumn{4}{|l|}{ WON size (cm): transverse axis } \\
\hline Mean (SD) & $10.2(4.6)$ & $10.7(6.8)$ & 0.784 \\
\hline Median & 9.2 & 7.8 & \\
\hline IQR & 5.6 & 7.1 & \\
\hline Range & $5.0-21.6$ & $5.0-32.0$ & \\
\hline \multicolumn{4}{|l|}{ WON size $(\mathrm{cm})$ : anteroposterior axis } \\
\hline Mean (SD) & $8.6(4.3)$ & $7.8(3.8)$ & 0.477 \\
\hline Median & 8.0 & 6.0 & \\
\hline IQR & 5.9 & 3.3 & \\
\hline Range & $2.4-20.0$ & $4.0-17.0$ & \\
\hline \multicolumn{4}{|l|}{ Route of drainage: $n(\%)$} \\
\hline Transesophageal & 0 & $1(3.4)$ & 0.509 \\
\hline Transgastric & $24(77.4)$ & $24(82.8)$ & \\
\hline Transduodenal & $7(22.6)$ & $4(13.8)^{*}$ & \\
\hline $\begin{array}{l}\text { Additional drainage tracts at } \\
\text { index procedure with nasocystic } \\
\text { catheter placement: } n(\%)\end{array}$ & $3(9.7)$ & $9(31.0)$ & 0.054 \\
\hline \multicolumn{4}{|l|}{ Pancreatic duct status: $\mathrm{n}(\%)$} \\
\hline Intact PD & $9(29.0)$ & $9(31.0)$ & 0.363 \\
\hline PD leak & $6(19.4)$ & $2(6.9)$ & \\
\hline DPDS & $13(41.9)$ & 17 (58.6) & \\
\hline Unknown & $3(9.7)$ & $1(3.4)$ & \\
\hline \multicolumn{4}{|l|}{ Procedure duration (min): } \\
\hline Mean (SD) & $18.0(15.5)$ & $41.6(25.7)$ & \\
\hline Median & 15 & 40 & $<0.001$ \\
\hline IQR & 17 & 28 & \\
\hline Range & $3-63$ & $10-115$ & \\
\hline Technical success: n (\%) & $31(100)$ & $29(100)$ & 0.999 \\
\hline
\end{tabular}

* One patient in the plastic stent group underwent drainage via both transgastric and transduodenal routes.

DPDS, disconnected pancreatic duct syndrome; LAMS, lumen-apposing metal stent; $\mathrm{PD}$, pancreatic duct; WON, walled-off necrosis.

3.2 procedures were required to achieve treatment success rate of $96.6 \%$ (table 3). Additional procedures undertaken due to suboptimal treatment response at 72 hours (for ongoing SIRS) included creation of additional transmural tracts to facilitate better drainage $(n=5)$, direct endoscopic necrosectomy $(n=6)$ and percutaneous drainage catheter placement $(n=5)$. The total number and details of individual procedures undertaken in the plastic cohort for treatment success until the 6-month follow-up period are shown in table 3 and online supplementary table 2, respectively.

Stent-related adverse events were encountered in two patients comprising migration of the stents into the jejunum in both patients. All migrated stents were retrieved at endoscopy using rat-tooth forceps. Clinical adverse events were encountered in four patients that were managed successfully. One patient presented with bleeding from the transmural tract; this patient bled from a previously treated splenic artery pseudoaneurysm 


\begin{tabular}{|c|c|c|c|}
\hline & LAMS $(n=31)$ & Plastic $(n=29)$ & $P$ values \\
\hline $\begin{array}{l}\text { Resolution of SIRS at } 24 \text { hours } \\
\text { post-treatment: } n(\%)\end{array}$ & $4(44.4)$ & $9(69.2)$ & 0.384 \\
\hline $\begin{array}{l}\text { Resolution of organ failure at } \\
24 \text { hours post-treatment: } n(\%)\end{array}$ & $1(50.0)$ & $1(25.0)$ & 0.999 \\
\hline Treatment success: $\mathrm{n}(\%)$ & $29(93.5)$ & $28(96.6)$ & 0.999 \\
\hline \multicolumn{4}{|l|}{ Length of hospital stay (days): } \\
\hline Mean (SD) & $6.2(9.0)$ & $12.2(21.1)$ & 0.129 \\
\hline Median & 3 & 4 & \\
\hline IQR & 6 & 13 & \\
\hline Range & $0-38$ & $0-103$ & \\
\hline \multicolumn{4}{|l|}{ Adverse events: $\mathrm{n}(\%)$} \\
\hline Overall & $13(41.9)$ & $6(20.7)$ & 0.077 \\
\hline Stent-related & $10(32.3)$ & $2(6.9)$ & 0.014 \\
\hline Prior protocol change & $8(25.8)$ & 0 & 0.005 \\
\hline After protocol change & $2(6.5)$ & $2(6.9)$ & 0.999 \\
\hline Clinical & $3(9.7)$ & $4(13.8)$ & 0.702 \\
\hline \multicolumn{4}{|c|}{ Total no. of procedures for treatment success: $\mathrm{n}(\%)$} \\
\hline Mean (SD) & $2.8(1.2)$ & $3.2(1.5)$ & 0.192 \\
\hline Median & 2 & 3 & \\
\hline IQR & 1 & 2 & \\
\hline Range & $2-7$ & $2-7$ & \\
\hline \multicolumn{4}{|l|}{ Total no. of readmissions: $\mathrm{n}(\%)$ * } \\
\hline 0 & $21(67.7)$ & $18(62.1)$ & 0.645 \\
\hline 1 & $8(25.8)$ & $9(31.0)$ & \\
\hline 2 & 0 & $2(6.9)$ & \\
\hline 3 & $2(6.5)$ & 0 & \\
\hline WON recurrence: $n$ (\%) & $1(3.2)$ & 0 & 0.999 \\
\hline \multicolumn{4}{|l|}{ Mean costs, 2017 US\$: } \\
\hline Total cost ${ }^{\dagger}$ & 53117 & 50132 & 0.775 \\
\hline Procedure cost & 12155 & 6609 & $<0.001$ \\
\hline
\end{tabular}

*From index procedure to last date of follow-up. The $p$ value infers to readmission vs no readmission.

†Total costs encompass costs for hospital stay, procedure, pharmacy, radiology, anaesthesia, laboratory and other support services.

LAMS, lumen-apposing metal stent; SIRS, systemic inflammatory response syndrome; WON, walled-off necrosis.

(prior to index intervention) and required a repeat coil embolisation. Respiratory events occurred in three patients that included aspiration pneumonia in two and pulmonary embolism in one.
Pancreatography revealed a normal main pancreatic duct in 9 , DPDS in 17, partial duct disruption in 2 and ductal status was unknown in 1 patient. A transpapillary pancreatic duct stent bridging the leak was placed in all patients with partial disruption, resulting in successful resolution of the leak. The indwelling plastic endoprostheses were left in situ indefinitely in all patients with DPDS.

\section{Outcomes at 6-month follow-up}

There was no significant difference in the total number of procedures needed to achieve treatment success between the two cohorts (table 3, online supplementary table 2), and the type of stent used did not affect the number of procedures performed (incidence rate ratio 1.12 (95\% CI 0.82 to 1.51$), p=0.478$ ), even when adjusted for possible clustering of procedures within the patients (online supplementary table 3 and 4). Also, there was no significant difference in the rate of clinical adverse events, treatment success, resolution of preintervention SIRS or LOS between the cohorts. While the overall stent-related adverse event rates were higher for LAMS $(32.3 \%$ vs $6.9 \%, p=0.014)$, there was no significant difference after the study protocol was amended $(6.5 \%$ vs $6.9 \%, p=0.999)$. The median procedure duration was significantly shorter for the LAMS cohort (median procedure duration $15 \mathrm{~min}$ for LAMS vs $40 \mathrm{~min}$ for plastic cohort, $\mathrm{p}<0.001$ ) and the procedure costs were significantly higher for LAMS when compared with plastic stents (table 3, online supplementary table 1). However, there was no significant difference in the total treatment costs between the groups.

At 6 months, there was no significant difference in the rate of readmissions $(32.3 \%$ vs $37.9 \%, \mathrm{p}=0.645)$ or recurrence (3.2\% vs $0 \%, p=0.999)$ between the LAMS and plastic stent cohorts, respectively (table 3 ). Readmissions were due to upper GI bleeding in four, LAMS migration resulting in gastric outlet obstruction in one, multiorgan failure from cirrhosis in one, abdominal pain/nausea/vomiting requiring conservative management in six and persistent WON requiring repeat intervention in five patients. Three patients were admitted with recurrent acute pancreatitis, resulting in death in two of these patients (one in each cohort). One patient in the LAMS cohort who had complete resolution of WON on CT at 6 weeks postintervention was admitted with recurrence of the WON at 3 months and elected to undergo surgical cystogastrostomy.

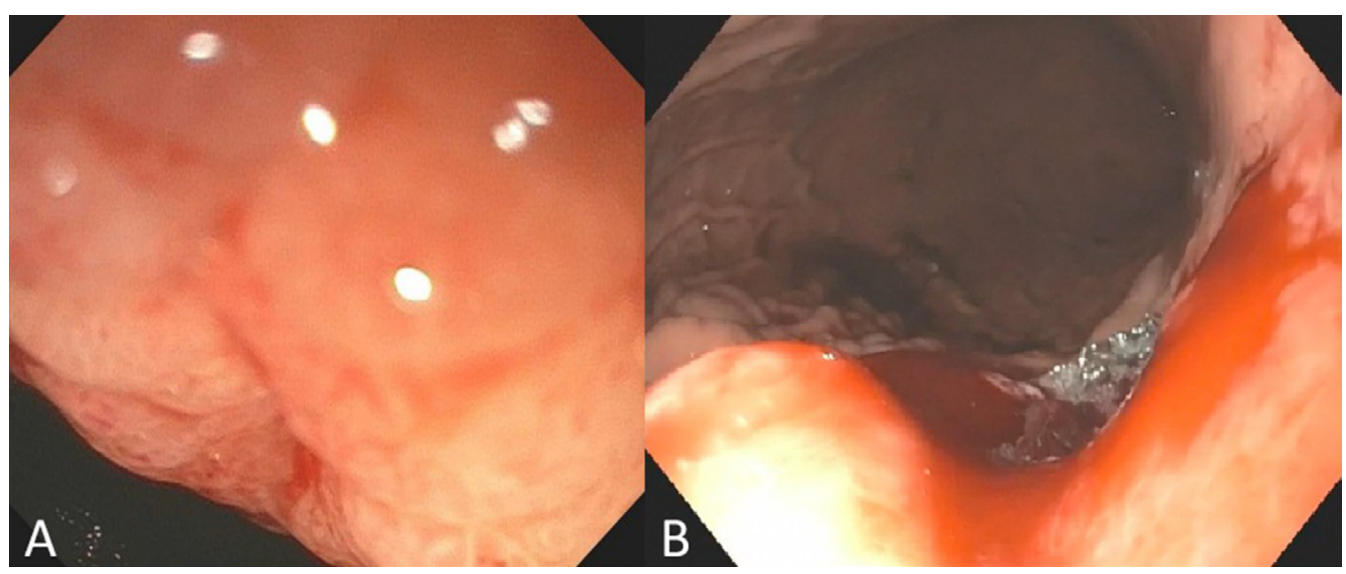

Figure 2 The lumen-apposing metal stents (LAMS) has completely migrated into the walled-off necrosis (WON) cavity and cannot be visualised on endoscopy (A). Endoscopic image of LAMS almost migrating inside the WON cavity (B). 


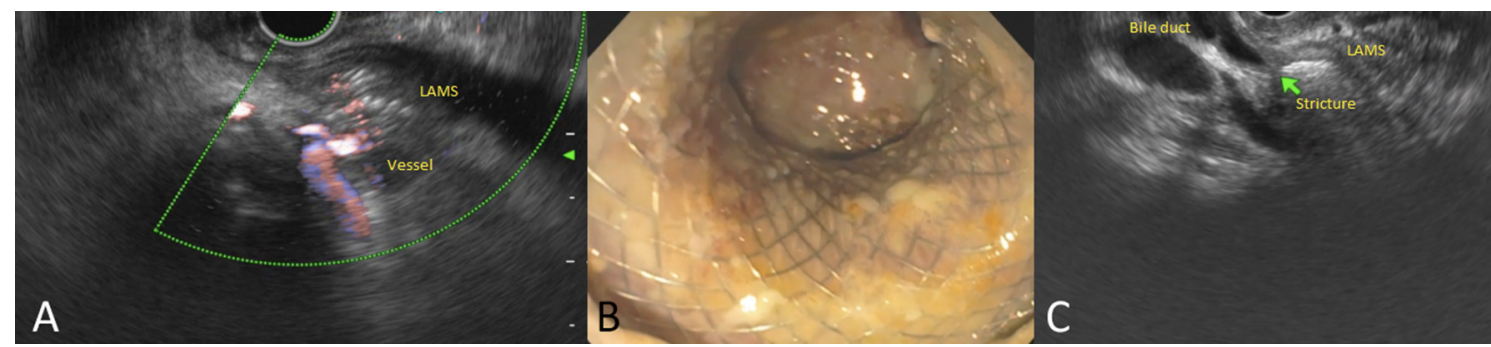

Figure 3 Endoscopic ultrasound (EUS) image of interlacing vessels within the distal flange of the lumen-apposing metal stents (LAMS) (A). Endoscopic image of LAMS, which was placed 5 weeks previously in the duodenal bulb (B). The LAMS can be seen causing biliary stricture on EUS due to external compression on the common bile duct (C).

\section{DISCUSSION}

In this study, we did not observe any significant difference in the primary or secondary outcomes between patients treated with LAMS or plastic stents except for initial procedure duration and the rate of stent-related adverse events prior to protocol change. Additionally, given the faster resolution of WON, to minimise adverse events, patients undergoing LAMS placement should undergo follow-up imaging at 3 weeks with stent removal if the fluid collection has resolved.

The rationale for using LAMS for WON drainage is that the larger diameter opening will provide faster and more complete drainage of the WON contents. Incomplete drainage can result in persistent symptoms and infection that may cause greater morbidity and require more interventions. In a retrospective, multicentre study that compared the efficacies of LAMS, plastic stent and fully covered self-expandable metal stent for drainage of WON, treatment success was lowest and number of reinterventions highest for patients treated with plastic stents. ${ }^{21}$ However, two recent retrospective studies observed no such difference in treatment outcomes except for the procedure duration, which was shorter when placing LAMS. ${ }^{11} 12$ Although it has been suggested that the procedure duration for performing reinterventions such as necrosectomy is shorter in patients with pre-existing LAMS, ${ }^{11}$ we observed no such difference: the median duration for reinterventions in the LAMS cohort was $107.5 \mathrm{~min}$ (IQR 42.5) vs $95 \mathrm{~min}$ (IQR 65) for plastic stents. Additionally, repeated passage of the endoscope through the LAMS oftentimes resulted in stent dislodgement (video 8). In such cases, placement of plastic stents was required to maintain patency of the fistulous tract.

We observed more stent-related adverse events, particularly bleeding in the LAMS cohort compared with some previously published retrospective studies. Likewise, a recent retrospective study reported a 19\% LAMS-associated bleeding in a cohort of 19 patients. ${ }^{22}$ We hypothesised that plastic stents likely gravitate towards the gut lumen as the WON resolves, whereas LAMS remains in place with the resultant friction between the stent and vasculature in the wall of the necrotic cavity precipitating bleeding. ${ }^{23}$ In a similar fashion, in patients undergoing transduodenal drainage, as the WON resolves, the LAMS can abut and compress the distal common bile duct causing obstructive jaundice. Additionally, by virtue of their lumen-apposing property, the stent may become deeply embedded in the gut wall resulting in tissue overgrowth. Historically, patients with PFCs were treated with plastic stents and have undergone follow-up imaging at 6 weeks to assess treatment response. ${ }^{19}$ In the present study, stent-related adverse events were observed $\geq 3$ weeks after LAMS placement or manifested at 6-week follow-up. We hypothesise that the wide diameter of the LAMS facilitates more rapid drainage of necrotic contents leading to faster WON resolution. However, once the WON resolves, the LAMS by virtue of its immobility, impinges on adjacent structures leading to bleeding, occlusion or becomes deeply embedded in the gut wall. Therefore, unlike in patients treated with plastic stents, we recommend a CT scan in 3 weeks followed by LAMS removal if the WON has resolved to minimise adverse events. We recommend validation of these observations by other investigators.

DPDS is an important but often overlooked complication, encountered in approximately $50 \%$ of patients with WON. In a recent study, we showed that the rate of PFC recurrence was significantly lower in patients with permanent indwelling transmural stents compared with patients in whom the stents were removed $(1.7 \%$ vs $17.4 \%, \mathrm{p}<0.001) .{ }^{24}$ Therefore, in clinical practice, we treat all patients with WON and suspected DPDS with plastic stents and leave them in situ indefinitely. In the present study, 13 of 31 patients in the LAMS cohort had DPDS; however, we were unable to exchange the LAMS for plastic stents in 6 of these 13 patients because the WON cavity had completely collapsed. One of these six patients presented with a $3 \mathrm{~cm}$ PFC, 8 months postintervention.

Four unmet needs should be addressed when evaluating a new technology for the treatment of WON: 1) achieve faster

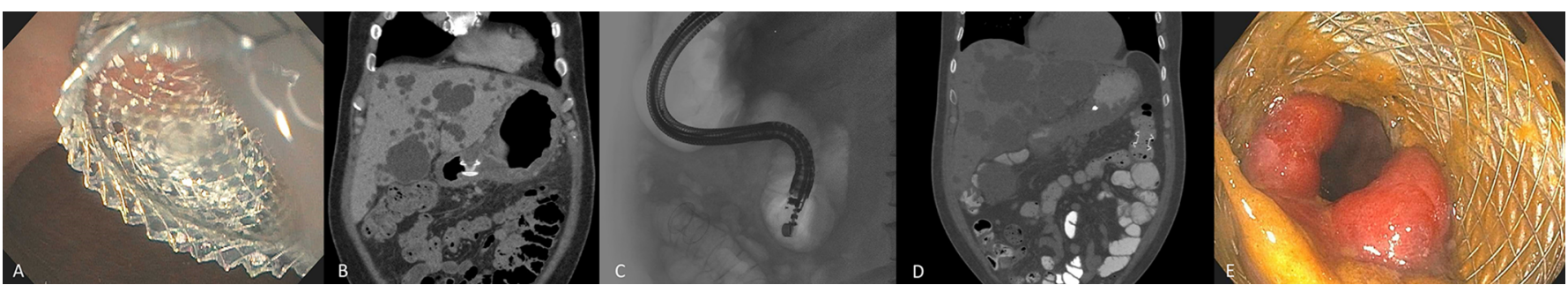

Figure 4 Lumen-apposing metal stents (LAMS) was placed via the transgastric route to drain the walled-off necrosis (A). The patient presented 16 days postprocedure with abdominal pain, where a CT showed that the LAMS had migrated out of the transmural tract and into the pylorus resulting in gastric outlet obstruction (B). The LAMS could no longer be seen in the gastric lumen on endoscopy as it had migrated further distally into the jejunum (C). Repeat CT showed that the LAMS had now migrated into the descending colon (D) and finally into the rectosigmoid junction (E), where it was removed via flexible sigmoidoscopy. 


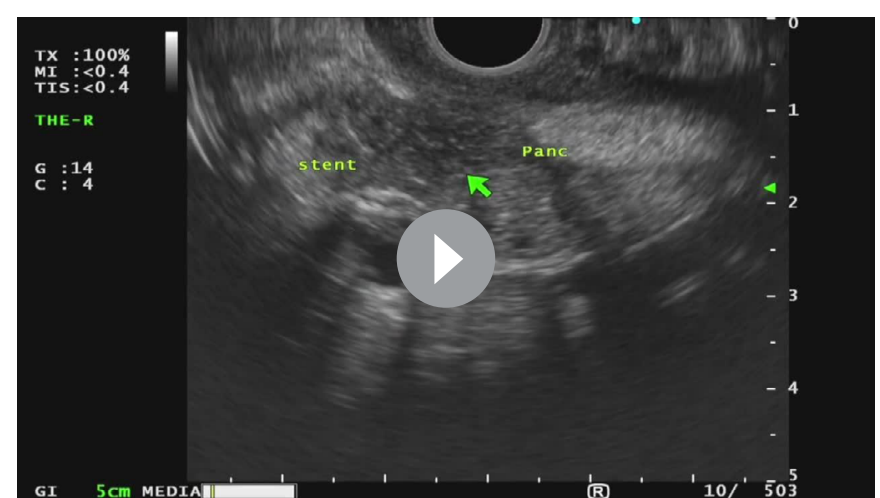

Video 7 Lumen-apposing metal stents (LAMS) cannot be exchanged for plastic stents in a patient with walled-off necrosis (WON) and disconnected pancreatic duct syndrome. EUS, endoscopic ultrasound.

resolution of WON with symptom relief, 2) decrease adverse events, 3) lower costs and 4) reduce the complexity of the procedure. In the present study, while LAMS was technically easier to place, the other needs were unmet. Although the wider opening of LAMS was expected to facilitate better drainage of necrotic contents, it did not translate into decreased number of procedures to achieve treatment success, higher resolution of pre-existing SIRS or shorter LOS. The outcomes of WON are dependent on several variables and transmural drainage is just one aspect of a multidisciplinary treatment strategy. The present study shows that while LAMS has some unique advantages, it does not 'fix' the underlying clinical problem. Also, the unique stent design does not appear to substantially improve the safety profile of the procedure; in fact, if the stents are left in situ for a prolonged period, there appears to be an increased risk of adverse events. We believe that our observations are very important given the increasing frequency with which LAMS are being used for other off-label indications such as enteral anastomosis, gallbladder drainage and lumen restoration procedures. ${ }^{25-27}$ Although the potential to lower costs would be a powerful rationale to apply to a new technology in WON drainage, the procedure-related costs were significantly higher with LAMS placement. The mean total costs were also higher by US $\$ 3000$ in the LAMS cohort, although this difference was not statistically significant.

Based on the findings of this randomised trial, the critical question is: which patients with WON are likely to benefit from LAMS rather than plastic stent placement? Given the technical ease, patients who might benefit from a shorter procedure duration are likely to benefit the most from LAMS placement. The use of LAMS must be avoided in patients who are unreliable to follow-up, with pseudoaneurysms in vicinity of WON (unless they are treated prior to LAMS placement), with suspected DPDS, and when cost is a major consideration. It is also important not to underestimate the importance of other adjunctive interventions such as creating additional transmural tracts or providing dual therapy by means of percutaneous drain placement (in addition to endoscopic transmural stent placement). These techniques, compared with endoscopic interventions delivered as a single therapy, improve clinical outcomes and decrease the need for aggressive measures such as necrosectomy. ${ }^{4} 19$

There are a few limitations to our study. One, we evaluated only a single design LAMS (Hot AXIOS) as other types LAMS are not commercially available in the USA. Two, some patients were treated using the multigate technique, which is not commonly

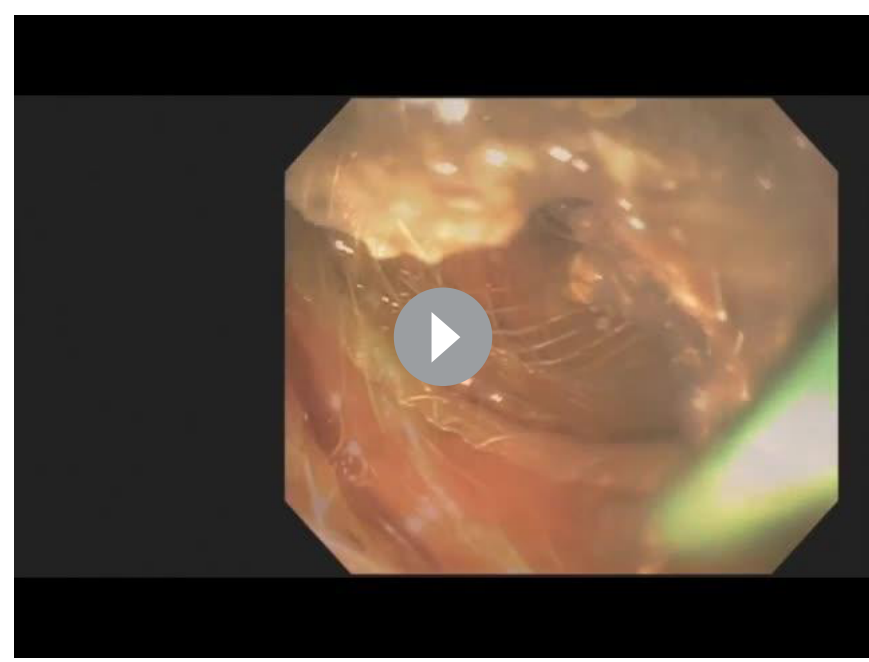

Video 8 Lumen-apposing metal stents (LAMS) becomes accidentally dislodged during endoscopic necrosectomy. EUS, endoscopic ultrasound; WON, walled-off necrosis.

practised at many centres. However, the technique facilitates better drainage of necrotic contents and obviates the need for high-risk interventions such as necrosectomy. Also, the need for additional interventions due to the underlying disease precludes straightforward comparison between the endoprostheses. Three, this was a single-centre study undertaken at a tertiary referral centre and procedures were performed by expert endoscopists, which may raise the concern of the generalisability of the trial results. However, the single-centre design strengthens the impact of the results because the treatment and follow-up protocol were standardised. Four, we did not report outcomes from a patient perspective and the primary outcome was not adjudicated in a blinded fashion. Finally, to limit the influence of percutaneous techniques on the study findings, we excluded patients with pre-existing drainage catheters prior to index intervention. Therefore, the applicability of the study findings in this patient subset is unclear.

In conclusion, except for procedure duration, we found no significant difference in clinical outcomes of patients with WON treated with LAMS or plastic stents. The choice of stent should be based on clinical status of the patient, presence of DPDS, likelihood of patient compliance with follow-up, multidisciplinary consensus and financial considerations when applicable.

Contributors JYB: study design, endoscopist performing procedures in the trial, acquisition of data, analysis and interpretation of data, statistical analysis, drafting of manuscript, critical revision of manuscript. SV: study concept and design, endoscopist performing procedures in the trial, interpretation of data, drafting of manuscript, critical revision of manuscript. BS: cost data analysis, drafting of manuscript, critical revision of manuscript. MKH, UN, RH: endoscopists performing procedures in the trial, critical revision of manuscript.

Funding The authors have not declared a specific grant for this research from any funding agency in the public, commercial or not-for-profit sectors.

Competing interests SV: Consultant for Olympus Medical Systems Corporation and Boston Scientific Corporation. RH: Consultant for Olympus Medical Systems Corporation and Boston Scientific Corporation.

Patient consent Obtained.

Ethics approval Institutional Review Board.

Provenance and peer review Not commissioned; externally peer reviewed.

Open access This is an open access article distributed in accordance with the Creative Commons Attribution Non Commercial (CC BY-NC 4.0) license, which permits others to distribute, remix, adapt, build upon this work non-commercially, 
and license their derivative works on different terms, provided the original work is properly cited, appropriate credit is given, any changes made indicated, and the use is non-commercial. See:http://creativecommons.org/licenses/by-nc/4.0/.

(c) Article author(s) (or their employer(s) unless otherwise stated in the text of the article) 2019. All rights reserved. No commercial use is permitted unless otherwise expressly granted.

\section{REFERENCES}

1 Varadarajulu S, Bang JY, Sutton BS, et al. Equal efficacy of endoscopic and surgical cystogastrostomy for pancreatic pseudocyst drainage in a randomized trial. Gastroenterology 2013;145:583-90.

2 Bang JY, Wilcox CM, Trevino JM, et al. Relationship between stent characteristics and treatment outcomes in endoscopic transmural drainage of uncomplicated pancreatic pseudocysts. Surg Endosc 2014;28:2877-83.

3 Baron TH, Harewood GC, Morgan DE, et al. Outcome differences after endoscopic drainage of pancreatic necrosis, acute pancreatic pseudocysts, and chronic pancreatic pseudocysts. Gastrointest Endosc 2002;56:7-17.

4 Varadarajulu S, Bang JY, Phadnis MA, et al. Endoscopic transmural drainage of peripancreatic fluid collections: outcomes and predictors of treatment success in 211 consecutive patients. J Gastrointest Surg 2011;15:2080-8.

5 Gluck M, Ross A, Irani S, et al. Dual modality drainage for symptomatic walled-off pancreatic necrosis reduces length of hospitalization, radiological procedures, and number of endoscopies compared to standard percutaneous drainage. J Gastrointest Surg 2012;16:248-57.

6 Varadarajulu S, Phadnis MA, Christein JD, et al. Multiple transluminal gateway technique for EUS-guided drainage of symptomatic walled-off pancreatic necrosis. Gastrointest Endosc 2011:74:74-80.

7 Gardner TB, Coelho-Prabhu N, Gordon SR, et al. Direct endoscopic necrosectomy for the treatment of walled-off pancreatic necrosis: results from a multicenter U.S. series. Gastrointest Endosc 2011;73:718-26.

8 Penn DE, Draganov PV, Wagh MS, et al. Prospective evaluation of the use of fully covered self-expanding metal stents for EUS-guided transmural drainage of pancreatic pseudocysts. Gastrointest Endosc 2012;76:679-84.

9 Gornals JB, De la Serna-Higuera C, Sánchez-Yague A, et al. Endosonography-guided drainage of pancreatic fluid collections with a novel lumen-apposing stent. Surg Endosc 2013;27:1428-34.

10 Itoi T, Binmoeller KF, Shah J, et al. Clinical evaluation of a novel lumen-apposing metal stent for endosonography-guided pancreatic pseudocyst and gallbladder drainage (with videos). Gastrointest Endosc 2012;75:870-6.

11 Mukai S, Itoi T, Baron TH, et al. Endoscopic ultrasound-guided placement of plastic vs. biflanged metal stents for therapy of walled-off necrosis: a retrospective single-center series. Endoscopy 2015;47:47-55.
12 Bang JY, Hasan MK, Navaneethan U, et al. Lumen-apposing metal stents for drainage of pancreatic fluid collections: When and for whom? Dig Endosc 2017;29:83-90.

13 Banks PA, Bollen TL, Dervenis C, et al. Classification of acute pancreatitis--2012: revision of the Atlanta classification and definitions by international consensus. Gut 2013;62:102-11

14 Arvanitakis M, Delhaye M, Bali MA, et al. Pancreatic-fluid collections: a randomized controlled trial regarding stent removal after endoscopic transmural drainage. Gastrointest Endosc 2007;65:609-19.

15 Manning WG, Mullahy J, O'Hagan A. Estimating log models: to transform or not to transform? J Health Econ 2001;20:461-94.

16 Pregibon D. Goodness of Link Tests for Generalized Linear Models. App/ Stat 1980;29:15-23.

17 Basu A, Rathouz PJ. Estimating marginal and incremental effects on health outcomes using flexible link and variance function models. Biostatistics 2005;6:93-109.

18 Cotton PB, Eisen G, Romagnuolo J, et al. Grading the complexity of endoscopic procedures: results of an ASGE working party. Gastrointest Endosc 2011;73:868-74.

19 Bang JY, Holt BA, Hawes RH, et al. Outcomes after implementing a tailored endoscopic step-up approach to walled-off necrosis in acute pancreatitis. Br J Surg 2014; 101:1729-38.

20 Belle $\mathrm{S}$, Collet $\mathrm{P}$, Post $\mathrm{S}$, et al. Temporary cystogastrostomy with self-expanding metallic stents for pancreatic necrosis. Endoscopy 2010;42:493-5.

21 Siddiqui AA, Kowalski TE, Loren DE, et al. Fully covered self-expanding metal stents versus lumen-apposing fully covered self-expanding metal stent versus plastic stents for endoscopic drainage of pancreatic walled-off necrosis: clinical outcomes and success. Gastrointest Endosc 2017;85:758-65.

22 Lang GD, Fritz C, Bhat T, et al. EUS-guided drainage of peripancreatic fluid collections with lumen-apposing metal stents and plastic double-pigtail stents: comparison of efficacy and adverse event rates. Gastrointest Endosc 2018;87. Epub ahead of print

23 Bang JY, Hasan M, Navaneethan U, et al. Lumen-apposing metal stents (LAMS) for pancreatic fluid collection (PFC) drainage: may not be business as usual. Gut 2017;66 Epub ahead of print.

24 Bang JY, Wilcox CM, Navaneethan U, et al. Impact of Disconnected Pancreatic Duct Syndrome on the Endoscopic Management of Pancreatic Fluid Collections. Ann Surg 2016:1. Epub ahead of print.

25 Itoi T, Baron TH, Khashab MA, et al. Technical review of endoscopic ultrasonographyguided gastroenterostomy in 2017. Dig Endosc 2017;29:495-502.

26 Dollhopf M, Larghi A, Will U, et al. EUS-guided gallbladder drainage in patients with acute cholecystitis and high surgical risk using an electrocautery-enhanced lumenapposing metal stent device. Gastrointest Endosc 2017;86:636-43. Epub ahead of print.

27 Irani S, Jalaj S, Ross A, et al. Use of a lumen-apposing metal stent to treat GI strictures (with videos). Gastrointest Endosc 2017;85:1285-9. 